\title{
Franck Salaün, Les Lumières. Une introduction
}

\section{Eszter Kovács}

\section{(2) OpenEdition}

\section{Journals}

\section{Édition électronique}

URL : http://journals.openedition.org/studifrancesi/3730

DOI : 10.4000/studifrancesi.3730

ISSN : 2427-5856

\section{Éditeur}

Rosenberg \& Sellier

\section{Édition imprimée}

Date de publication : 1 décembre 2012

Pagination : $564-565$

ISSN : 0039-2944

\section{Référence électronique}

Eszter Kovács, «Franck Salaün, Les Lumières. Une introduction », Studi Francesi [En ligne], 168 (LVI | III) | 2012, mis en ligne le 30 novembre 2015, consulté le 06 mars 2021. URL : http://

journals.openedition.org/studifrancesi/3730; DOI : https://doi.org/10.4000/studifrancesi.3730

Ce document a été généré automatiquement le 6 mars 2021.

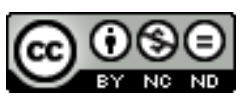

Studi Francesi è distribuita con Licenza Creative Commons Attribuzione - Non commerciale - Non opere derivate 4.0 Internazionale. 


\title{
Franck Salaün, Les Lumières. Une introduction
}

\author{
Eszter Kovács
}

\section{RÉFÉRENCE}

FRANCK SALAÜN, Les Lumières. Une introduction, Paris, PUF, (coll. Licence Lettres), 2011, pp. 265.

1 Nous connaissons tous le souci - parfois la peine - d'introduire un cours, un article, une communication, un livre. Introduire quelque chose peut paraître plus difficile que de le développer ou de le détailler. Or, l'objectif du livre de Franck Salaün est d'introduire les étudiants du premier cycle dans l'étude des Lumières. Il va de soi que le domaine à examiner est si vaste qu'il serait impossible de faire plus dans un seul ouvrage. L'auteur précise dans l'avant-propos qu'il conçoit ce livre un peu comme un cours, comme un dialogue entre l'enseignant et les étudiants et essaie de considérer leurs remarques, leurs questions, leurs hésitations éventuelles. En rédigeant cet ouvrage, il s'est sans aucun doute servi à la fois de ses expériences de chercheur et d'enseignant.

2 L'essentiel d'une introduction est de poser certaines questions à partir d'un certain nombre de constats. Le choix de Franck Salaün est de considérer les Lumières comme un phénomène complexe: une période de la philosophie et de la littérature européennes avec des objectifs communs, sans être unifiée, qui aura un impact jusqu'à nos jours. Le livre se compose de cinq parties qui correspondent à autant d'approches du sujet. Dans le premier chapitre, intitulé «Un siècle philosophe», Salaün essaie de délimiter (et ainsi définir) les Lumières: il s'interroge sur les sens du mot, sur la notion, sur son acception depuis le XVIII ${ }^{\mathrm{e}}$ siècle et sur son rôle dans un contexte plus large que les Lumières françaises. Il distingue les Lumières au sens large et au sens stricte du terme; ce dernier correspond grosso modo à la période de 1750 à 1780 . Il affirme que la réclamation de la liberté de penser est à l'origine du phénomène, qui apparait, de ce point de vue, non pas comme un simple courant mais comme un débat. Il consacre un 
développement relativement plus long aux sujets dont il est spécialiste, comme le matérialisme. Il ne considère pas la Révolution comme l'aboutissement des Lumières, les principales figures des Lumières en France étant mortes avant 1789 - il reviendra sur cette question dans la conclusion. Cette opinion, contredisant une représentation devenue "classique», est justifiable si on regarde les Lumières comme un phénomène difficile à saisir, qui ne se limite pas à certains «mots d'ordre».

Le chapitre «Les Lumières au sens stricte» s'occupe entre autres sujets de l'Encyclopédie, de son enjeu et de son impact. Salaün examine également la figure du philosophe: il le définit comme un acteur de la vie publique, qui ose penser par lui-même et qui est ainsi souvent en conflit avec les autorités. Le chapitre présente finalement «quelques acteurs» - Voltaire, Diderot, D'Alembert, d'Holbach, Beccaria, Rousseau, Malesherbes et Rey - non pas leur biographie à proprement parler ou leur œuvre mais leur rôle dans la période en question. Naturellement, il s'agit d'un aperçu de quelques pages consacrées à chacun.

4 Le chapitre «Une configuration singulière» commence par l'analyse du texte De l'horrible danger de la lecture de Voltaire, une facétie qui démontre par l'ironie, voire par l'absurde, que la liberté de penser est inaliénable de l'homme. Salaün décrit ensuite le fonctionnement de la censure et de l'opinion publique. Il élargit dans la suite la période en question en considérant l'œuvre de certains prédécesseurs. À côté de deux auteurs qui sont à part - Montesquieu et Voltaire - il s'occupe de Fontenelle, de Bayle, de Locke. La pensée des Lumières apparaît donc à la fois comme une innovation et une continuation.

Dans le chapitre «Quelques thèmes essentiels», il parle de certaines préoccupations de la littérature des Lumières: l'apparition de la production du livre dans la littérature, le souci de l'origine (ou des origines), la figure du philosophe dans la fiction et dans le théâtre et la question du jugement (préjugés versus opinion juste). Le chapitre «Formes et styles typiques» commence par une réflexion sur la hiérarchie des genres, et examine plus en détail les genres qui traduisent les questionnements majeurs des Lumières. Parmi ces genres «représentatifs», il consacre quelques pages au dialogue d'idées, à la parodie, au genre sérieux et aux articles de dictionnaires.

6 L'auteur rappelle dans la conclusion, intitulée «L'avenir d'un débat», que la relation entre les Lumières et la Révolution n'est pas un simple rapport de cause à effet, contrairement à une image très fortement enracinée, soit qu'elle valorise, soit qu'elle rejette les Lumières. Il examine plus en détail certaines idées reçues pour démontrer qu'elles sont trompeuses, en tout cas réductrices: par exemple la représentation des Lumières comme une doctrine commune et unifiée doit être contestée. Il s'interroge finalement sur l'actualité des Lumières.

7 Quant à la réussite de l'ouvrage comme un outil d'enseignement, il faut certainement attendre la réaction du public auquel il est destiné. L'auteur essaie d'adapter son texte à cet objectif - la lecture ne demande pas de connaissances préalables trop spécialisées, les commentaires sont faciles à suivre. Il se trouve trois tableaux récapitulatifs et treize «encadrés» dans le livre - il s'agit d'extraits relativement longs, de données (par exemple sur l'édition de l'Encyclopédie) ou d'illustrations un peu particulières (par exemple une fiche relative à l'emprisonnement de Diderot). On trouve une bibliographie sélective à la fin de chaque chapitre et un glossaire à la fin du volume. communs. Franck Salaün essaie d'éviter ce piège justement en allant à l'envers: il veut 
nuancer certaines généralités ou réfuter certains lieux communs, certaines représentations partiales. Philosophie et littérature sont liées dans cette approche, comme elles l'étaient à l'époque des Lumières. Les sujets qu'il aborde concernent certains aspects des Lumières, mais c'est l'ambition même de l'ouvrage. 